\title{
In Vitro Culture of Immature Zygotic Mango Embryos and Plantlet Development
}

\author{
Juan Bernardo Pérez-Hernández and María José Grajal-Martín ${ }^{1}$ \\ Departamento de Fruticultura Tropical, Instituto Canario de Investigaciones \\ Agrarias, Apartado 60, La Laguna-38200, Santa Cruz de Tenerife, Spain
}

Additional index words. breeding, embryo culture, Mangifera indica, tissue culture

\begin{abstract}
In vitro culture of immature embryos may assist mango breeding in the production of hybrid plant material. However, zygotic embryo culture techniques have not been successfully developed for mango. To recover in vitro zygotic plants through embryo culture, 'Lippens' and 'Keitt' were used as a source of model immature embryos. Excised embryos were incubated in a liquid maturation medium to test different culture systems and media composition. Subsequent germination allowed for the recovery of complete in vitro plantlets. Variables included during artificial embryo maturation, independently or through paired interactions, significantly affected all the parameters measured for embryo development and characterization of the plantlets. Main effects of culture system (i.e., static versus agitation) and coconut water supply (20\%) were responsible for up to $85.5 \%$ of total treatment variation. Direct and inverse interactions observed between culture system and either coconut water supplement or sucrose content (45 or $60 \mathrm{~g} \cdot \mathrm{L}^{-1}$ ) contributed to define the best combination of factors to improve embryo growth and plant formation. Complete plantlets could be obtained at a frequency above $83 \%$ for both cultivars at the end of the in vitro phase at a developmental stage that allowed acclimatization to greenhouse conditions.
\end{abstract}

The mango is an important fruit crop cultivated throughout the tropics and subtropics. Despite ranking fifth in total world fruit production (FAO, 2010), its breeding potential has not been as well exploited as that of other major crops. Most of the commercial cultivars available today are chance seedlings derived from open, or more rarely controlled, pollinations (Iyer and Schnell, 2009). Breeders are not only limited by constraints common to many fruit tree species (long juvenility, selfincompatibility, high heterozygosity, and low seed production), but are further hampered by a number of specific drawbacks, i.e., 1) extremely low hand-pollination efficiency, ranging frequently between $0.1 \%$ and $1 \%$ (Iyer and Schnell, 2009); 2) poor fruit set, usually less than 1\% (Usman et al., 2001); and 3) severe natural fruit drop that causes premature loss of many of the scarce fruits derived from putative successful hand crosses (Bally et al., 2009). Therefore, in vitro culture of immature embryos has been long considered useful given its potential to increase the recovery rate of hybrid plant material for subsequent evaluation in mango breeding programs (Iyer and

Received for publication 2 June 2011. Accepted for publication 6 Sept. 2011.

The study, included in research project RTA2006-182, was financed by the Spanish Ministry of Science and Innovation and FEDER funds.

We thank Sandra Petit (lab assistance), María del Carmen Cid (statistical analysis), and Tina Redard (language revision).

${ }^{1}$ To whom reprint requests should be addressed; e-mailmjgrajal@icia.es.
Subramanyam, 1971). It would also offer an advance to the recovery of interploid crosses that, in vivo, generally fail to complete their development.

Although literature on mango somatic embryogenesis abounds (recently reviewed by Krishna and Singh, 2007; Litz et al., 2009) and embryo rescue has been successfully applied in plant breeding for raising hybrids in other species (Sharma et al., 1996), little exists on the culture of mango zygotic embryos. Probable reasons are the generalized recalcitrance of mango tissues derived from adult plants to in vitro procedures, in which the chief obstacles are the inherently slow in vitro response and phenolic oxidation giving rise to media browning and, more importantly, explant necrosis (Krishna et al., 2008). Recent experience gained in the culture of somatic embryos could be used now for the successful culture of zygotic mango embryos. In this context, Pateña et al. (2002) effectively controlled browning of nucellar explants by adding coconut water to the embryogenesis induction medium. In addition, maturation of somatic embryos is routinely carried out on solid medium, although culture under liquid medium agitation has been shown to improve elongation of underdeveloped somatic embryos (DeWald et al., 1989b). With regard to sucrose content, different concentrations have been used for the maturation of mango somatic embryos, but reports on its influence on zygotic embryo maturation are absent.

The aim of the present study was to establish a protocol for in vitro plant recovery through embryo culture and test its performance on zygotic embryos from well-known mango cultivars during maturation under different culture systems and growth media.

\section{Materials and Methods}

Fertilized ovules derived from openpollinated trees were aseptically excised from disinfected $3.5 \pm 0.5$-cm long fruitlets, between 30 to $45 \mathrm{~d}$ after anthesis, and used to extract the endosperm and the immature embryos. Liquid endosperm was collected by combining extractions from enough 'Lippens' and 'Keitt' fruitlets to complete four $50-\mu \mathrm{L}$ samples and measuring total soluble solids (TSS) using a refractometer (Atago PAL-1, Tokyo, Japan). Embryos between 4 and $11 \mathrm{~mm}$ in length of monoembryonic mango cultivars Lippens and Keitt were established in a maturation medium containing B-5 major salts (Gamborg et al., 1968, as modified by DeWald et al., 1989b), Murashige and Skoog (MS) minor salts and organics (Murashige and Skoog 1962), glutamine $(2.74 \mathrm{~mm})$, and casein hydrolisate $(0.025 \%$ w/v), adapted from DeWald et al. (1989a). To this basal media, trial treatments included adding sucrose (45 or $60 \mathrm{~g} \cdot \mathrm{L}^{-1}$ ) and coconut water (CW) (Sigma-Aldrich, St. Louis, MO) $(0 \%$ or $20 \% \mathrm{v} / \mathrm{v}$ ) and applying two different liquid culture systems, static and agitated.

Static culture was done on saturated paper bridges in test tubes containing $15 \mathrm{~mL}$ of medium during a 4-week period. Agitated culture took place over $21 \mathrm{~d}$, with weekly subcultures, in six-well titer plates containing $5 \mathrm{~mL}$ of medium under continuous shaking at $40 \mathrm{rpm}$. The incubation process was done in the dark. All the development phases described subsequently were carried out under a 16-h light photoperiod.

Embryo germination was done over a 4-week period on a solid germination medium consisting of half-strength B-5 major salts, MS minor salts and vitamins, $20 \%(\mathrm{v} / \mathrm{v})$ $\mathrm{CW}, 0.025 \%(\mathrm{w} / \mathrm{v})$ casein hydrolysate, 2.74 mM glutamine, $30 \mathrm{~g} \cdot \mathrm{L}^{-1}$ sucrose, and $0.75 \%$ Bacto-agar (DeWald et al., 1989a). Germinated embryos were then cultured in liquid regeneration medium composed of B-5 major salts, MS minor salts and vitamins, $2.74 \mathrm{~mm}$ glutamine, and $30 \mathrm{~g} \cdot \mathrm{L}^{-1}$ sucrose (Ara et al., 1999). Plantlet development was carried out during two cycles of $14 \mathrm{~d}$ under these conditions. The $\mathrm{pH}$ of all media was adjusted to 5.8 before autoclaving.

For acclimatization, in vitro-produced plants were transferred to $10.5-\mathrm{cm}$ diameter plastic pots containing peat as substrate and cultured in the greenhouse under a mist system.

The experiment was arranged in a factorial design with individually cultured embryos considered as experimental units. The experiment was repeated twice, during two consecutive fruiting seasons, each treatment consisting of four to 12 replicates to include a total of 90 and 92 embryos for 'Keitt' and 'Lippens', respectively. Embryo growth rate was determined at the end of the maturation phase and calculated as a ratio between final and initial longitudinal size; length of total root (primary and secondary), shoot, and longest leaf as well as dichotomous ( 0 or 1$)$ 
score for complete plant formation, were determined at the end of the plantlet development phase.

Quantitative results were subjected to partitioning of treatment sum of squares for statistical analysis, according to Little (1981). Categorical results were analyzed through logistic regression. Percentage of total treatment variation for significant factors and interactions affecting quantitative variables as well as odd ratio for categorical variables was calculated using SPSS 15.0 software (SPSS Inc., Chicago, IL). The level of significance was in all cases established at 5\% probability.

\section{Results and Discussion}

The sequence of in vitro development from immature zygotic embryo to plantlet formation is illustrated in Figure 1. Excised zygotic embryos were at an early cotyledonary stage (Fig. 1A). Earlier in development, embryos at the torpedo stage are still translucent and fragile, easily damaged during extraction. During these initial phases of fruit growth, the zygotic embryo is completely immersed in a liquid endosperm that serves as nourishing broth for its development (Sturrock, 1967). Emulating these natural conditions, the liquid-phase medium was likewise used during initial maturation to test performance of embryos, because previous tests on solid medium resulted in severe tissue blackening (not shown). Under liquid incubation, on the other hand, both static and agitated culture systems (Fig. 1B-E) promoted embryo growth and consequent complete in vitro plantlet formation (Fig. 1F-H), allowing the ex vitro acclimatization of plants (Fig. 1I-K).

Partitioning the treatment sum of squares shows the relative relevance of significant single factors and interactions for total treatment variation (Table 1 ). Considering the culture system as an independent statistical factor, it accounted for $54.6 \%$ to $85.5 \%$ of total treatment variation (Table 1). This effect, although common to both cultivars, is illustrated in Figure 2 for 'Lippens' material, showing that agitation of cultures during maturation doubled embryo growth and increased four- to sevenfold the size of the root, shoot, and longest leaf in recovered plants. Whereas maturation of somatic embryos is mostly carried out on solid media, as reported in the literature, DeWald et al. (1989b) also obtained better elongation rates when using agitated culture to achieve maturation of heart-shaped, 3- to 5-mm long in vitro-raised somatic embryos. In our case, liquid culture, with or without agitation, did not avoid either tissue necrosis or medium browning, but exudation of phenols during maturation was more evident in the static culture system, because these compounds accumulated in the supporting filter paper (Fig. 1D). Although some tissue browning will normally occur on the surface of embryos in agitated cultures, probably as a result of rub damage (Fig. 1E), this culture system appears to have reduced exudation and browning and may, therefore, be responsible for the improved in vitro

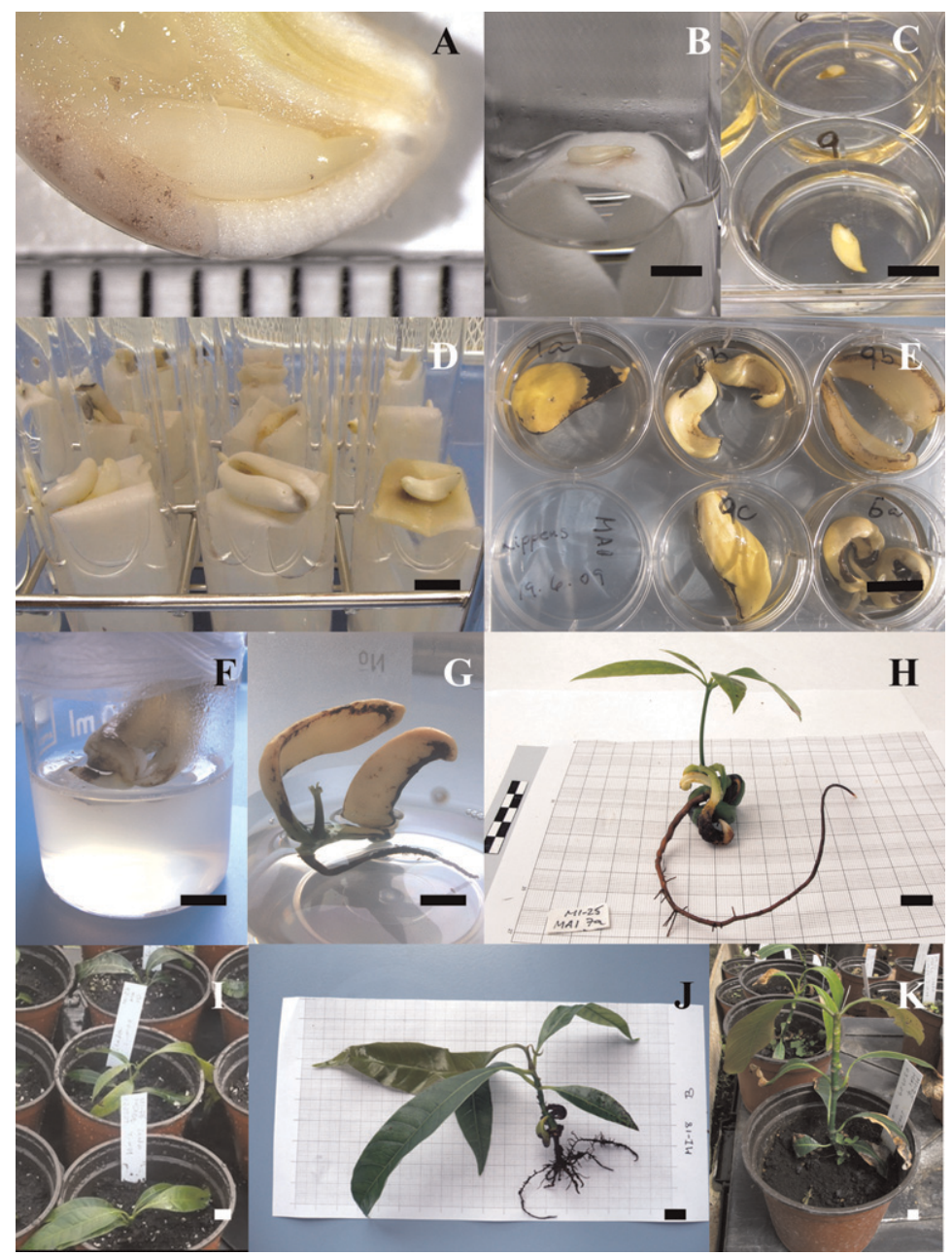

Fig. 1. In vitro plantlet development through embryo rescue. (A) Dissected fertilized ovule showing a 5-mm-long immature embryo (scale in $\mathrm{mm}$ ). (B) Static incubation of excised embryos on liquid medium-saturated paper bridges in test tubes. (C) Agitated culture of excised embryos in six-well titer plates. (D) Embryos after maturation in static culture. (E) Embryos after maturation in agitated culture. (F) Embryo germination showing root emission and first leaves. (G) Elongation of the shoot-root axis in development medium. (H) Complete plantlet obtained at the end of the in vitro phase (12 weeks after culture initiation). (I) Acclimatization of plantlets in the greenhouse at Day 0. (J) 'Lippens' seedling (derived from an 8-mm long embryo maturated in agitated liquid medium containing $45 \mathrm{~g} \cdot \mathrm{L}^{-1}$ sucrose and coconut water) after $58 \mathrm{~d}$ under acclimatization conditions. (K) Acclimatizated 'Keitt' seedling after 7 months (originally from a 9-mm long embryo maturated under agitation in medium with $60 \mathrm{~g} \cdot \mathrm{L}^{-1}$ sucrose and coconut water) (bars $\mathbf{B}-\mathbf{C}=8 \mathrm{~mm}$; $\mathbf{D}-\mathbf{K}=10 \mathrm{~mm}$ ).

Table 1. Partitioning of treatment sum of squares. ${ }^{2}$

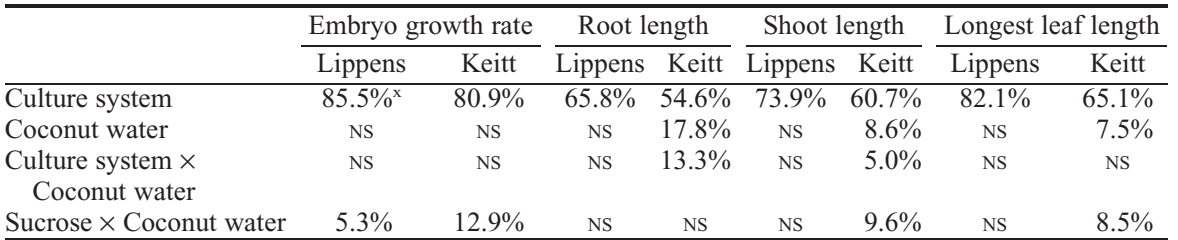

${ }^{\text {zPercent }}$ of total treatment variation for factors and interactions with significant effect on different variables $^{y}$ for mango cvs. Lippens and Keitt.

${ }^{y}$ Embryo growth rate as determined at the end of embryo development; root, shoot, and longest leaf length as measured after plant formation.

${ }^{x}$ Significant at $P \leq 0.05$.

NS = non-significant.

growth of embryos during maturation (Fig. 2). In addition, agitated liquid culture increases the contact surface of the explant with the medium and favors the diffusion of nutrients, gases, and toxic metabolites. Under these conditions, the initial better performance of embryos resulted in a drastic improvement of all other plantlet parameters (Fig. 2).

Sucrose concentrations as low as $20 \mathrm{~g} \cdot \mathrm{L}^{-1}$ and as high as $60 \mathrm{~g} \cdot \mathrm{L}^{-1}$ are generally used in media for the maturation of mango embryos derived from somatic embryogenesis (DeWald 
et al., 1989b; Pateña et al., 2002). In planta, however, where the liquid endosperm provides endogenous sugars, determination of its TSS content resulted closer to this upper limit, ranging between 5.7 and $6.6^{\circ} \mathrm{Brix}$. We therefore restricted sucrose variation tests to the mentioned concentrations of 45 and $60 \mathrm{~g} \cdot \mathrm{L}^{-1}$. At these levels and as an independent factor, sucrose did not play a significant role in zygotic embryo maturation or in any of the other parameters quantified during plantlet development for either cultivar (Table 1). On the other hand, the effect of CW, either independently or interacting with the culture system or sucrose concentration, affected all variables in 'Keitt' embryos (Table 1). Although significant, its contribution to the variation among treatments was limited, ranging between $5.0 \%$ and $17.8 \%$ to reveal a minor effect (Table 1). The main effect of the presence of $\mathrm{CW}$ in the maturation medium was observed on the marginal means for the root, shoot, and longest leaf (Fig. 3) following a pattern similar to that of the culture system (Fig. 2). CW also participated in all paired interactions detected (Table 1). Either under static or agitated culture, or at the lower sucrose content, the addition of $\mathrm{CW}$ enhanced embryo growth for the two cultivars as well as increased root, shoot, and longest leaf length in 'Keitt'. Figure 4 illustrates different patterns for these interactions. Addition of CW promoted embryo growth at low sucrose concentration and was detrimental at the higher sugar content. This inverse effect on embryo growth was practically identical in both cultivars (Fig. 4A-B), making the combined use of $20 \% \mathrm{CW}$ and $45 \mathrm{~g} \cdot \mathrm{L}^{-1}$ sucrose significantly raise 'Lippens' and 'Keitt' embryo growth rates to $\approx 3.20$ and 4.10 , respectively. It could be hypothesized that whereas at lower sucrose content, the addition of $\mathrm{CW}$ might be beneficial by supplying extra growth factors and reducing explant browning, adverse effects observed at a higher sucrose concentration could relate to the resulting osmotic potential of the media. A similar inverse interaction between these two variables was also found to affect shoot and longest leaf length in 'Keitt' material (not shown). Conversely, a direct interaction between the presence of CW and culture system was observed to influence 'Keitt' root and shoot length (Figs. 4C and D, respectively). Addition of CW markedly enhanced the positive effect of agitated incubation of embryos to increase total root length and shoot size of in vitro-recovered plants in $\approx 60$ and $7 \mathrm{~mm}$, respectively.

Statistically, the recovery frequency for complete plants was affected only by the main effect of the culture system. Complete plants were recovered at frequencies above $83 \%$ in both cultivars when embryo maturation was carried out in agitation (Fig. 5). In addition, logistic regression analysis revealed that, compared with static culture, agitated incubation of immature embryos increases approximately six and 10 times the probability to obtain a complete plant of 'Lippens' and 'Keitt', respectively (Fig. 5).

In vitro-produced plantlets were transferred to ex vitro conditions to preliminarily test their acclimatization capacity (Fig. 1I).

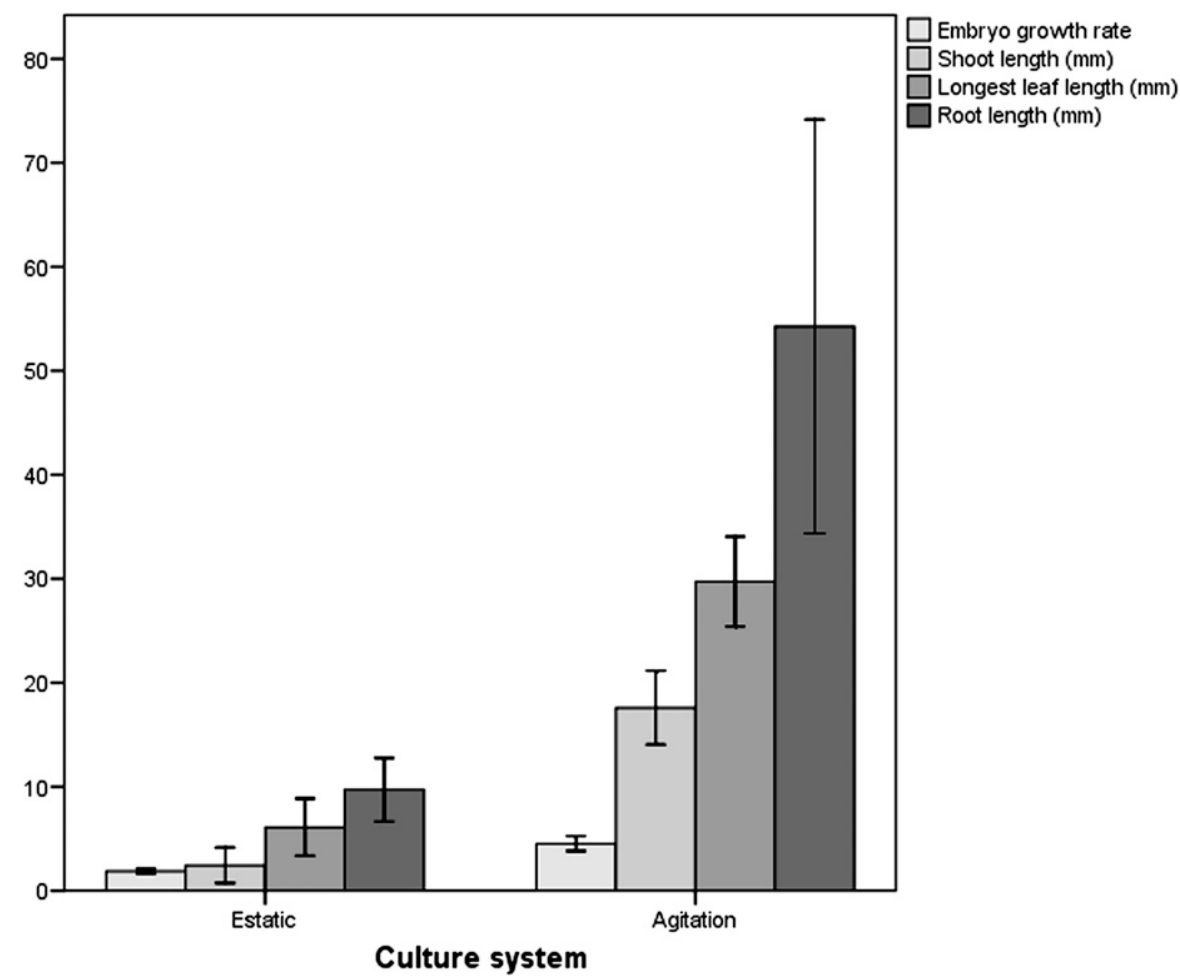

Fig. 2. Marginal means for the main effect of culture system on embryo growth rate (determined at the end of embryo development), shoot, longest leaf, and root length (measured after plant formation) in 'Lippens' material. Error bars represent 95\% confidence interval.

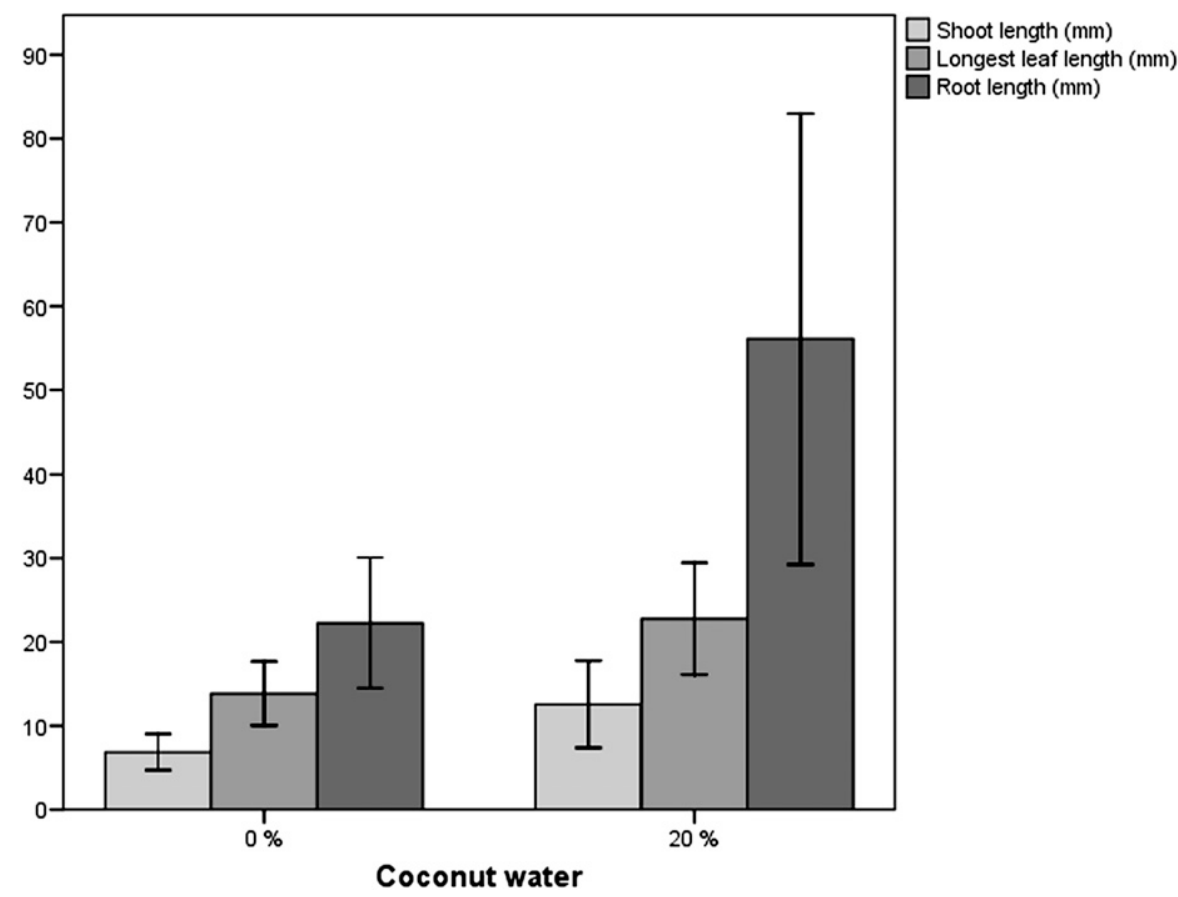

Fig. 3. Marginal means for the main effect of coconut water on shoot, longest leaf, and root length (measured after plant formation) in 'Keitt' material. Error bars represent 95\% confidence interval.

Although acclimatization studies are not concluded at the time of writing this article, plants derived from embryos matured under improved artificial conditions have shown competence to successfully develop a branched root system and complete the expansion of new leaves emitted ex vitro (Fig. 1J). Conse- quently, the benefit of agitated liquid incubation on embryo growth, enhanced by addition of $\mathrm{CW}$ and low sucrose content, not only raised the recovery probabilities and the final quality of the in vitro plants, but also allowed ex vitro survival of the plantlets produced. Therefore, the inclusion of an artificial maturation phase 

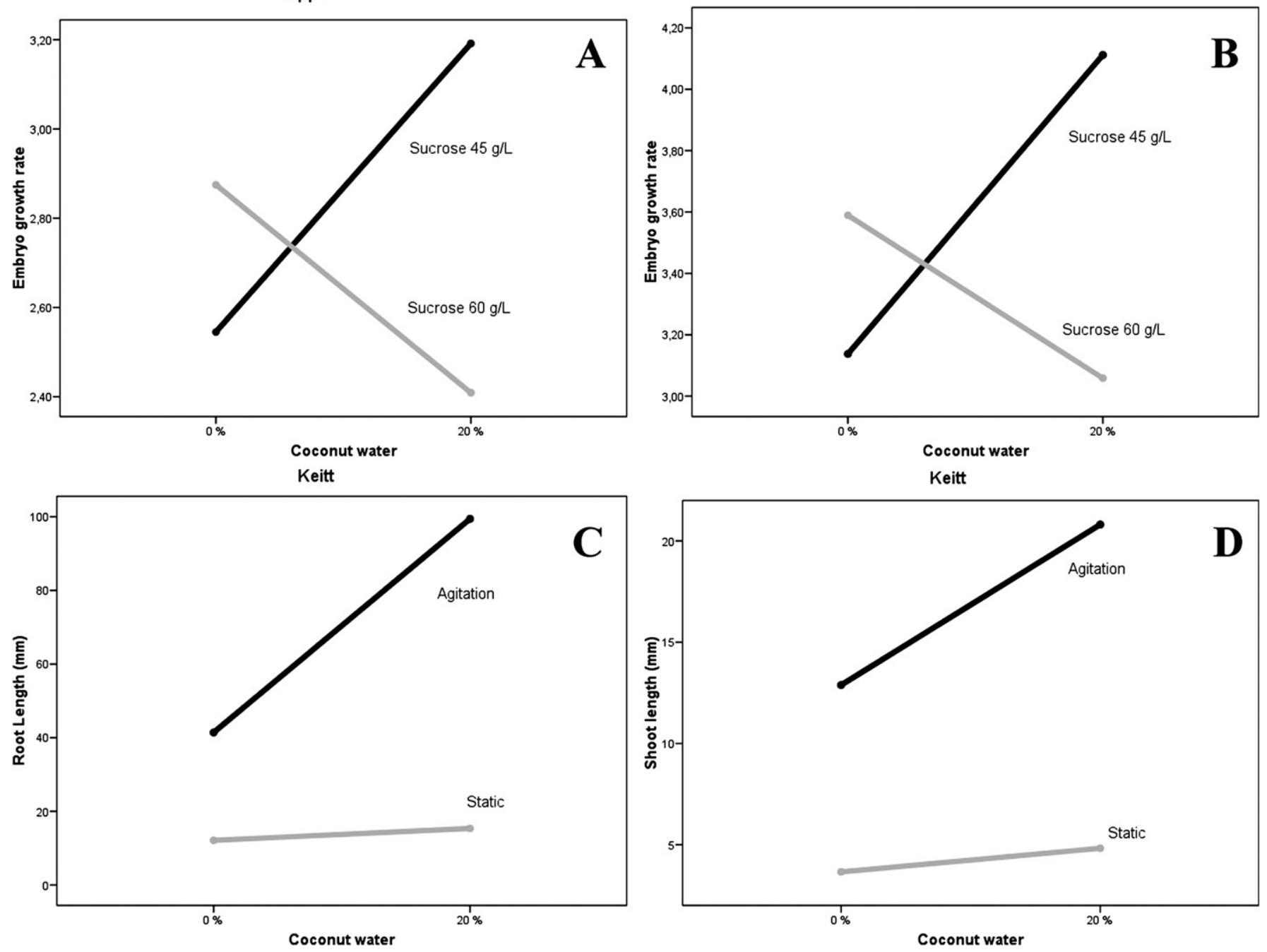

Fig. 4. Marginal means for the interacting effect of sucrose $\times$ coconut water on embryo growth rate (determined at the end of embryo development) in 'Lippens' (A) and 'Keitt' (B) and for the interacting effect of culture system $\times$ coconut water on root $(\mathbf{C})$ and shoot (D) length (measured after plant formation) in 'Keitt' material.

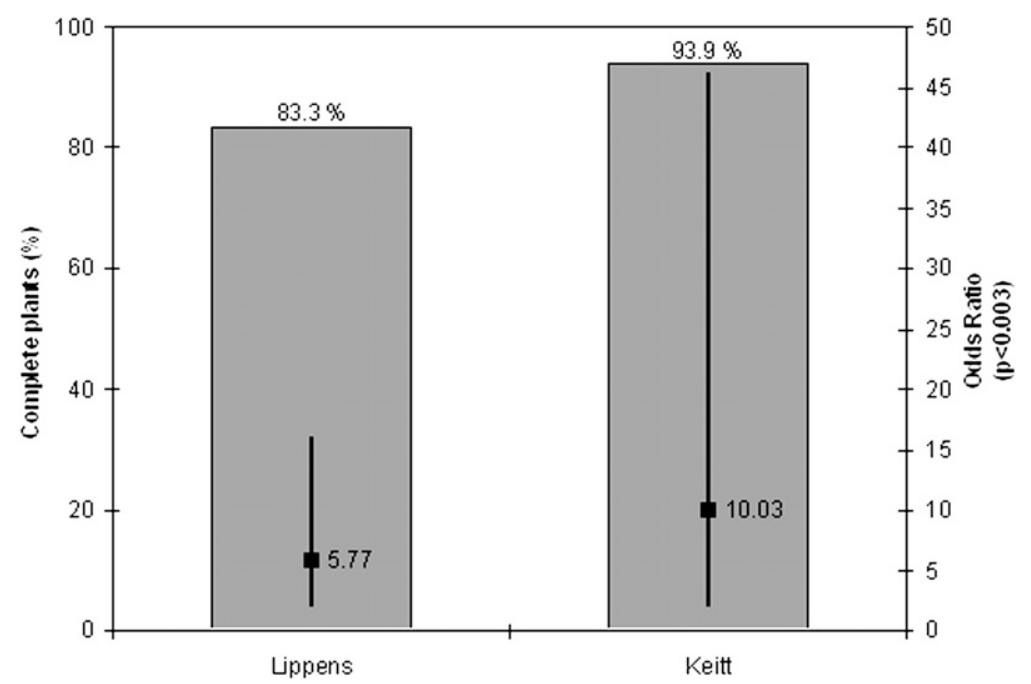

Fig. 5. Main effect of culture system on the formation of complete in vitro plants: percentage of complete plants and odds ratio for complete plant formation from 'Lippens' and 'Keitt' immature embryos cultured under agitation. Error bars represent $95 \%$ confidence interval. might additionally be of critical importance to this final aim. Indeed, $100 \%$ mortality has been reported in mango when in vitrorecovered plants derive from precociously germinated immature embryos (Sahijram et al., 2005, 2009)

The present study shows that agitated incubation during embryo maturation was advantageous for the developing embryo. The effects were carried over in the subsequent in vitro growth of the plantlets of both cultivars and increased the probability for obtaining complete plants. In addition, low sucrose content in a maturation medium supplemented with $\mathrm{CW}$ favored the development of the embryo and could further promote the in vitro growth of the plantlets. This combination of culture system and medium supplements is therefore proposed for the initial culture of immature mango embryos followed by germination and plantlet formation. The whole procedure takes $\approx 3$ months of culture to obtain complete plants at the end of the in vitro phase. The technique might be a valuable tool for hybrid embryo culture to assist mango breeding programs. 


\section{Literature Cited}

Ara, H., U. Jaiswal, and V.S. Jaiswal. 1999. Germination and plantlet regeneration from encapsulated somatic embryos of mango (Mangifera indica L.). Plant Cell Rpt. 19:166-170.

Bally, I.S.E., P. Lu, and P.R. Johnson. 2009. Mango breeding, p. 51-82. In: Jain, S.M. and P.M. Priyadarshan (eds.). Breeding plantation tree crops: Tropical species. Springer Science + Business Media, New York, NY.

DeWald, S.G., R.E. Litz, and G.A. Moore. 1989a. Optimizing somatic embryo production in mango. J. Amer. Soc. Hort. Sci. 114:712-716.

DeWald, S.G., R.E. Litz, and G.A. Moore. 1989b. Maturation and germination of mango somatic embryos. J. Amer. Soc. Hort. Sci. 114:837-841.

FAO. 2010. Statistical database. 15 Feb. 2011.<http:// faostat.fao.org $>$.

Gamborg, O.L., R.A. Miller, and K. Ojima. 1968. Plant cell cultures: I. Nutrient requirements of suspension cultures of soybean root cells. Exp. Cell Res. 50:151-158.
Iyer, C.P.A. and R.J. Schnell. 2009. Breeding and genetics, p. 67-96. In: Litz, R.E. (ed.). The mango: Botany, production and uses. $\mathrm{CAB}$ International, Wallingford, UK.

Iyer, C.P.A. and M.D. Subramanyam. 1971. Possible role of embryo culture on mango breeding. Indian J. Hort. 29:135-136.

Krishna, H., R.K. Sairam, S.K. Singh, V.B. Patel, R.R Sharma, M. Grover, L. Nain, and A. Sachdev. 2008. Mango explant browning: Effect of ontogenic age, mycorrhization and pre-treatments. Sci. Hort. 118:132-138.

Krishna, H. and S.K. Singh. 2007. Biotechnological advances in mango (Mangifera indica L.) and their future implication in crop improvement-A review. Biotechnol. Adv. 25:223-243.

Little, T.M. 1981. Interpretation and presentation of results. HortScience 16:19-22.

Litz, R.E., M.A. Gómez-Lim, and U. Lavi. 2009. Biotechnology, p. 641-669. In: Litz, R.E. (ed.). The mango: Botany, production and uses. $\mathrm{CAB}$ International, Wallingford, UK.
Murashige, T. and F. Skoog. 1962. A revised medium for rapid growth and bioassays with tobacco tissue culture. Physiol. Plant. 15:473-497.

Pateña, L.F., L.R. Carlos-Refuerzo, and R.C. Barba. 2002. Somatic embryogenesis and plantlet regeneration in mango (Mangifera indica L.). In Vitro Cell. Dev. Biol. Plant 38:173-177.

Sahijram, L., K.T. Bollamma, A. Naren, J.R. Soneji, M.R. Dinesh, and G.K. Halesh. 2005. In vitro hybrid embryo rescue in mango (Mangifera indica L.) breeding. Indian J. Hort. 62:235-237. Sahijram, L., M.R. Dinesh, Y.T.N. Reddy, J.R. Soneji, K.T. Bollamma, Y. Sreevalli, and A. Naren. 2009. Ex vitro shoot-tip grafting for rescuing hybrid vitro plants of mango (Mangifera indica L.) developed through embryo culture. Acta Hort. 820:319-322.

Sharma, D.R., R. Kaur, and K. Kumar. 1996. Embryo rescue in plants - A review. Euphytica 89:325-337.

Sturrock, T. 1967. Nucellar embryos of the mango. Proc. Fla. State Hort. Soc. 80:350-354.

Usman, M., B. Fatimaand, and M.J. Jaskani. 2001. Breeding in mango. Intl. J. Agr. Biol. 3:522-526. 